\title{
Knockdown of B7H6 inhibits tumor progression in triple-negative breast cancer
}

\author{
BING ZHANG, JINZHONG SUN, XIAOLI YAO, JUANJUAN LI, YI TU, FENG YAO and SHENGRONG SUN \\ Department of Breast and Thyroid Surgery, Renmin Hospital of Wuhan University, Wuhan, Hubei 430060, P.R. China
}

Received April 28, 2017; Accepted January 22, 2018

DOI: $10.3892 / 01.2018 .8689$

\begin{abstract}
The B7 family, the most common family of secondary signaling molecules, consists of eight cell-surface proteins, which regulate the $\mathrm{T}$-cell mediated immune response by delivering co-inhibitory or co-stimulatory signals through their corresponding ligands. Among them, natural killer cell cytotoxicity receptor 3 ligand 1 (NCR3LG1, also known as B7H6) has been reported as a new member, and is involved in tumor progression of various types of human cancer. However, the role of B7H6 in triple-negative breast cancer (TNBC) remains unknown. In the present study, western blotting was performed to determine the protein expression levels of B7H6 in a normal mammary epithelial cell line (MCF-10A), non-TNBC breast cancer cell lines (MCF-7 and AU565) and TNBC cell lines (MDA-MB-231 and MDA-MB-468). B7H6 was knocked down using small interfering RNA, and an MTT assay was performed to determine proliferation ability, flow cytometry was used to analyze apoptosis, and Transwell and wound-healing assays were performed to measure migration ability. Expression of proliferation-associated proteins (SMAD family member 4 and $\beta$-catenin) and apoptosis-associated proteins (BCL2 associated X, BCL2 apoptosis regulator and caspase-3) were analyzed by western blotting. The results demonstrated that B7H6 was highly expressed in TNBC cells, and that knockdown of B7H6 inhibited cell proliferation and migration, and promoted apoptosis. Furthermore, the results revealed that proliferation and apoptosis-associated proteins were altered in the B7H6-knockdown MDA-MB-231 cells. In conclusion, the present study demonstrated that B7H6 may have significant roles in the regulation of cell proliferation, apoptosis and migration of TNBC cells.
\end{abstract}

Correspondence to: Dr Shengrong Sun, Department of Breast and Thyroid Surgery, Renmin Hospital of Wuhan University, 236 Jiefang Road, Wuhan, Hubei 430060, P.R. China

E-mail: sunshengrong6523@126.com

Key words: B7 family, natural killer cell cytotoxicity receptor 3 ligand 1, triple-negative breast cancer, tumor progression

\section{Introduction}

Breast cancer (BC) is one of the most common types of cancer worldwide, accounting for $20-23 \%$ of total cancer cases, and the incidence is increasing each year $(1,2)$. Although the diagnostic techniques and therapeutic strategies have improved over the past decades, $\mathrm{BC}$ remains the leading cause of cancer death in females worldwide (3).

Triple-negative breast cancers (TNBCs), accounting for $\sim 25 \%$ of BC cases, are characterized by tumors that lack expression of the estrogen receptor (ER), progesterone receptor (PR) and the human epidermal growth factor receptor 2 (HER2) (4,5). Because the majority of basal-like cancers are also ER-, PR-, and HER2-negative ( $80 \%)$, it has been suggested that the triple-negative and basal-like phenotypes are effectively synonymous, but data from immunohistochemical analysis have revealed that this is not the case (6-8). Triple-negative is a phenotype that encompasses more than one molecular subtype: its major components are the basal-like, normal, and claudin-low molecular subtypes, including BRCA1-deficient breast tumors (9). Hormone receptor-positive breast tumors are treated with endocrine manipulations, such as tamoxifen and aromatase inhibitors (10). However, patients with TNBC cannot benefit from endocrine therapy because of the lack of ER, PR, and HER2 expression. The current treatment options for TNBC are limited, and include surgery, radiation therapy, and systemic treatment. Chemotherapy is currently the only approved systemic treatment for TNBC, which is frequently accompanied by considerable side effects $(1,11)$. Therefore, there is an urgent need to investigate the molecular mechanisms and to explore novel diagnostic and therapeutic strategies for TNBC.

The B7 family of proteins consists of co-stimulatory or co-inhibitory molecules that are expressed mainly on the cell surface of antigen presenting cells and serve a key role in the regulation of the immune system $(12,13)$. Among the B7 family, natural killer cell cytotoxicity receptor 3 ligand 1 (NCR3LG1, also known as B7H6) was identified as a tumor-specific ligand for the natural cytotoxicity triggering receptor 3 (NCR3) (14). It has been reported that B7H6 is expressed in various types of tumor cells, including lung, ovarian and renal cell cancer, but not expressed in normal tissues $(14,15)$. Thus, B7H6 holds therapeutic potential for cancer, but its exact role in cancer is not fully elucidated yet. In specific, the mechanisms by which B7H6 may affect tumor progression have not been identified fully in TNBC cells. 
In the present study, it was hypothesized that knockdown of B7H6 might be involved in inhibiting tumor progression of TNBC cells. Suppression of B7H6 could be used as a potential target for treatment of TNBC. Therefore, the role of B7H6 in cell viability, apoptosis and migration of TNBC cells was investigated.

\section{Materials and methods}

Cell culture. Human normal mammary epithelial cell line (MCF-10A), BC cell lines (MCF-7, AU565), and TNBC cell lines (MDA-MB-231, MDA-MB-468) were all obtained from the American Tissue Cell Culture collection (ATCC; Manassas, VA, USA). MCF-10A cells were maintained in Dulbecco's modified Eagle's medium (DMEM)/F12 medium (Invitrogen; Thermo Fisher Scientific, Inc., Waltham, MA, USA) supplemented with $10 \mu \mathrm{g} / \mathrm{ml}$ insulin (Sigma-Aldrich; Merck KGaA, Darmstadt, Germany), 100 ng/ml cholera enterotoxin, $0.5 \mu \mathrm{g} / \mathrm{ml}$ hydrocortisol, and $20 \mathrm{ng} / \mathrm{ml}$ epidermal growth factor (EGF). MCF-7 and AU565 cells were cultured in RPMI 1640 (Invitrogen; Thermo Fisher Scientific, Inc.) with $10 \%$ fetal bovine serum (FBS; Sigma-Aldrich; Merck KGaA). MDA-MB-231 and MDA-MB-468 cells were propagated in DMEM/H-21 (Invitrogen; Thermo Fisher Scientific, Inc.) with $10 \%$ FBS. All cells were cultured in a humidified $37^{\circ} \mathrm{C}$ incubator with $5 \% \mathrm{CO}_{2}$.

Small interfering (si) RNA transfection. siRNAs were commercially designed and purchased from Thermo Fisher Scientific, Inc. The sequence of the siRNA for B7H6 was 5'-CGGCAC AGTCTTTCTGAAACT-3'. Cells were transfected with $50 \mathrm{nM}$ scramble siRNA (negative control, or NC sense: 5'-UUCUCC GAACGUGUCACGUTT-3', antisense: 5'-ACGUGACAC GUUCGGAGAATT-3') or B7H6-siRNA with Lipofectamine 3000 (Thermo Fisher Scientific, Inc.), according to the manufacturer's protocol. After $6 \mathrm{~h}$, the transfection medium (Opti-MEM; Gibco; Thermo Fisher Scientific, Inc.) was removed and the cells were maintained in normal medium for an additional $24 \mathrm{~h}$. Western blot assay was performed to assess the transfection efficiency.

Western blot analysis. Cells were washed with cold PBS and treated with cell lysis buffer (Cell Signaling Technology, Inc., Danvers, MA, USA) at $4^{\circ} \mathrm{C}$ or on ice for $2 \mathrm{~h}$. Total proteins were prepared using the Extraction kit (Beyotime Institute of Biotechnology, Shanghai, China), and the concentration was determined by a BCA Protein assay kit (Bio-Rad Laboratories, Inc., Hercules, CA, USA). Then proteins (40 $\mu \mathrm{g})$ were separated by SDS-PAGE and transferred to polyvinylidene fluoride membrane (Bio-Rad Laboratories, Inc.). To block the nonspecific signals, membranes were incubated with $5 \%$ low fat milk for at least $2 \mathrm{~h}$ at room temperature. Then membranes were incubated with primary antibody overnight at $4^{\circ} \mathrm{C}$. The membranes were then washed with $5 \%$ bovine serum albumin in TBS/0.1\% Tween-20 (TBS/T), and incubated with a horseradish peroxidase-conjugated secondary antibody (1:1,000; ab205718; Abcam, Cambridge, MA, USA) for $2 \mathrm{~h}$ at $37^{\circ} \mathrm{C}$. The results were visualized using the enhanced chemiluminescence substrate kit (Amersham; GE Healthcare, Chicago, IL, USA). The protein quantity was analyzed by Image Lab software (version 5.0; Bio-Rad, Hercules, CA, USA). The antibodies used in the present study were: anti-GAPDH (1:1,000; Santa Cruz Biotechnology, Inc., Dallas, TX, USA), anti-SMAD family member 4 (SMAD4; 1:300; ab40759; Abcam, Cambridge, MA, USA), anti- $\beta$-Catenin $(1: 1,000 ;$ ab32572; Abcam), anti-BCL2 associated X (Bax; 1:1,000; ab32503; Abcam), anti-BCL2 apoptosis regulator (Bcl-2; ab32124; 1:1,000; Abcam), and anti-Caspase-3 (1:1,000; ab13585; Abcam).

Cell viability assay (MTT assay). MTT assay was performed in order to evaluate the effects of B7H6 siRNA on TNBC cell lines, as previously described (16). Briefly, cells were harvested with trypsin and seeded at a concentration of $3 \times 10^{3}$ cells/well into a 96-well plate. Then, $20 \mu \mathrm{l}$ of MTT reagent was added to each well, and incubated at $37^{\circ} \mathrm{C}$ for $4 \mathrm{~h}$, followed by the addition of $150 \mu \mathrm{l}$ dimethyl sulfoxide. Finally, absorbance was determined by using a microplate reader at $490 \mathrm{~nm}$ (Sunrise; Tecan Group Ltd., Männedorf, Switzerland) at 48 h.

Flow cytometry analysis. Cell apoptosis was investigated by flow cytometry using Annexin V/propidium iodide (PI) double staining (BD Biosciences, Franklin Lakes, NJ, USA). The cells were plated onto 6-well plates at a concentration of $1 \times 10^{6}$ cells/well. Following incubation for $24 \mathrm{~h}$, cells were washed twice with cold PBS, and resuspended in $500 \mu 1$ binding buffer containing $5 \mu 1$ fluorescein isothiocyanate (FITC)-labeled Annexin V and $5 \mu \mathrm{l}$ PI for at least $10 \mathrm{~min}$ at room temperature in the dark. Finally, cells were analyzed by flow cytometry and data were analyzed using Cell Quest Pro software (BD Biosciences).

Transwell assay. The cell migration assay was performed in 6-well Transwell chambers with $8-\mu \mathrm{m}$ pore size polycarbonate membranes (Corning Inc., Corning, NY, USA). Cells were suspended in serum-free medium at a density of $1 \times 10^{5}$ cells/well, then $100 \mu 1$ of this cell suspension was seeded in the upper chamber. The lower chamber was filled with $500 \mu 1$ medium supplemented with $20 \%$ fetal calf serum (Gibco; Thermo Fisher Scientific, Inc.). Following $24 \mathrm{~h}$ of incubation at $37^{\circ} \mathrm{C}$ in $5 \% \mathrm{CO}_{2}$, cells on the upper surface of the membrane were removed, and the lower surface of the inserts was fixed in methanol for $10 \mathrm{~min}$ and stained with crystal violet. The cells that had migrated to the lower surface were photographed and measured using an inverted phase contrast microscope (magnification, x40; Olympus Corporation, Tokyo, Japan).

Wound-healing assay. Cells were seeded in 6-well plates at the density of $1.5 \times 10^{5}$ cells/well, and cultured overnight until confluent. Then the cells were treated with siRNAs (50 nM negative control siRNA or $50 \mathrm{nM}$ B7H6 siRNA) for $48 \mathrm{~h}$. A straight scratch was made through the confluent cells with a pipette tip. Cells were washed twice with PBS, and images (magnification, x100) were captured immediately as baseline. Fresh medium was then added and images of the same location were captured at $48 \mathrm{~h}$. The ratio of the remaining wound area relative to the initial wound area was calculated using Image-Pro Plus software (version 6.0; Media Cybernetics, Bethesda, MD, USA). 

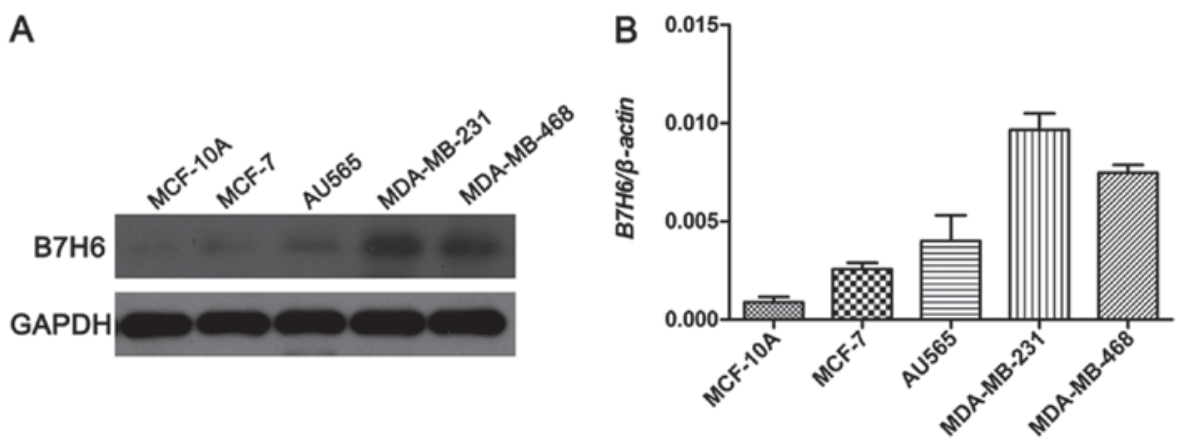

Figure 1. Expression levels of B7H6 in breast cancer cell lines. (A) Western blot assay was performed to detect the protein expression levels of B7H6 in a normal mammary epithelial cell line (MCF-10A), a ER+/PR+ breast cancer cell line (MCF-7), a HER2+ breast cancer cell line (AU565), and two triple-negative breast cancer cell lines (MDA-MB-231 and MDA-MB-468). GAPDH was used as the internal standard. (B) Quantitative data analysis. B7H6, natural killer cell cytotoxicity receptor 3 ligand 1 .

Statistical analysis. Data are presented as mean \pm standard deviation. SPSS 21.0 software (IBM Corp., Armonk, NY, USA) and GraphPad (GraphPad Software, Inc., La Jolla, CA, USA) were used to conduct the analysis. Statistical differences between two groups were examined with unpaired Student's t-test and multiple groups were compared using one-way analysis of variance followed by Tukey's post hoc test. $\mathrm{P}<0.05$ was considered to indicate a statistically significant difference.

\section{Results}

B7H6 is highly expressed in TNBC cell lines. To investigate the potential biological roles of B7H6 in TNBC, the expression levels of B7H6 were examined using western blot analysis in a normal mammary epithelial cell line (MCF-10A), a ER+/PR+ BC cell line (MCF-7), a HER2+ BC cell line (AU565), and two TNBC cell lines (MDA-MB-231 and MDA-MB-468). Among these, the results demonstrated that B7H6 levels were markedly higher in the MDA-MB-231 and MDA-MB-468 cell lines compared to MCF-10A, MCF-7 and AU565 cell lines (Fig. 1).

B7H6 knockdown inhibits cell proliferation and promotes apoptosis in TNBC cells. To further assess the biological function of B7H6 in TNBC cells, the expression of B7H6 was silenced in MDA-MB-231 cells, and the effects of B7H6 silencing on cell proliferation were examined using the MTT assay. First, the efficiency of the B7H6-specific siRNA was confirmed by western blotting. The results demonstrated that transfection of the MDA-MB-231 cells with the B7H6 siRNA resulted in a marked downregulation of B7H6 expression compared with control (Fig. 2A). The results from the MTT assay indicated that knockdown of B7H6 significantly inhibited the proliferation of MDA-MB-231 cells compared with cells transfected with negative control siRNA (Fig. 2B). Apoptosis is a significant aspect of cancer cell progression, so to determine whether apoptosis could be induced by B7H6 siRNA, annexin V/PI double staining was performed followed by flow cytometry analysis. The results demonstrated that the $\%$ of apoptotic cells significantly increased following transfection with B7H6 siRNA compared with control cells (Fig. 2C).

B7H6 knockdown suppresses cell migration in MDA-MB-231 cells. To validate the effects of B7H6 on cell migration,
Transwell and wound-healing assays were conducted in MDA-MB-231 cells transfected with B7H6 or negative control siRNA. Results from the Transwell assay demonstrated that cell migration was significantly decreased in the B7H6 knockdown group compared with the control group (Fig. 3A). In the wound-healing assay, the scratch healed slower in B7H6-knockdown MDA-MB-231 cells compared with the control group (Fig. 3B), suggesting that B7H6 inhibits cell migration in TNBC cells.

B7H6 knockdown upregulates the expression of $\beta$-catenin, caspase-3, and Bax, and downregulates the expression of SMAD4 and Bcl-2 in TNBC cells. To further confirm the effects of B7H6 on cell proliferation and apoptosis, the expression of several proliferation and apoptosis-related proteins was examined in TNBC cells transfected with B7H6 siRNA. Western blot analysis revealed that SMAD4 expression was downregulated and $\beta$-catenin expression was upregulated following B7H6 knockdown, compared with cells transfected with negative control siRNA (Fig. 4), indicating that B7H6 promoted proliferation in TNBC cells. In addition, the results demonstrated that knockdown of B7H6 increased the production of caspase- 3 and $\mathrm{Bax}$, and decreased the production of Bcl-2 in TNBC cells (Fig. 4), suggesting that B7H6 inhibited TNBC cell apoptosis.

\section{Discussion}

Despite an overall decreased mortality of TNBC, the incidence appears to be on the rise and the prognosis remains poorer than the other types of BC (17). Given the lack of molecular targets, traditional treatments have limited effect, and the possibility of metastasis of TNBC to visceral sites, including the brain, is higher than the other subtypes (18). Recent studies have demonstrated that EGF receptor (EGFR) and vascular endothelial growth factor (VEGF) are involved in the progression of TNBC cells in vitro (19). It was reported that bevacizumab, an anti-angiogenic agent that targets VEGF, could induce a significant improvement in progression-free survival of TNBC (20). Similar reports have suggested that EGFR is expressed on approximately half of the basal-like $\mathrm{BC}$ cells, and is indispensable for the proliferation of basal-like cell lines $(21,22)$. Recently, an antibody against 

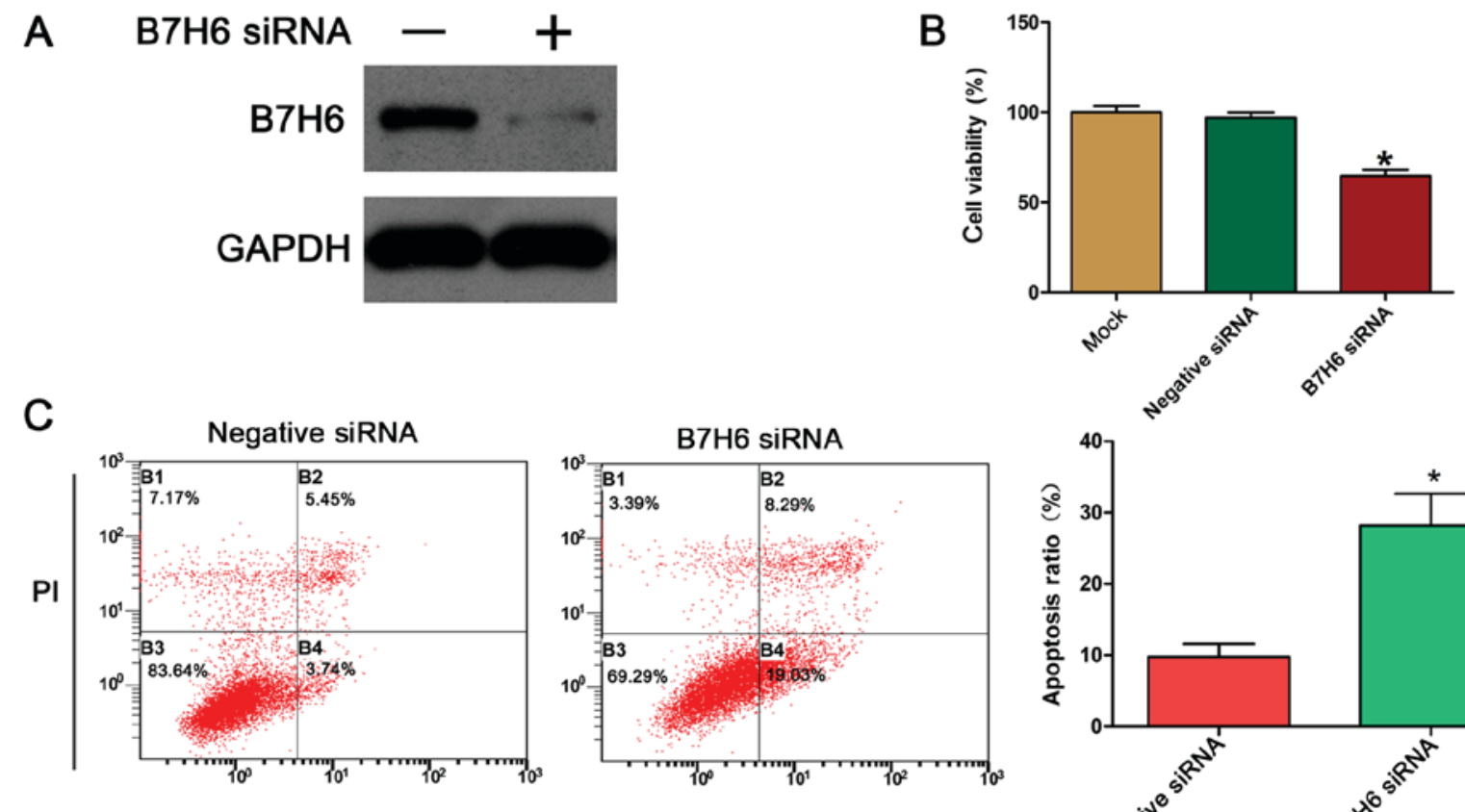

Annexin V

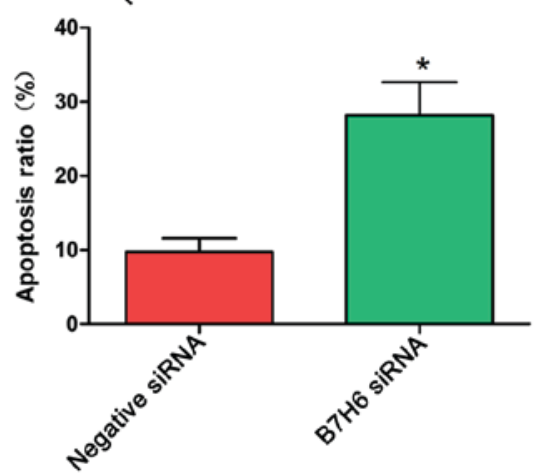

Figure 2. Silencing of B7H6 by siRNA inhibits proliferation and promotes apoptosis in breast cancer cells. (A) Western blot assay was performed to measure the knockdown efficiency of B7H6 siRNA. GAPDH was used as the internal standard. (B) Cell viability was detected by the MTT assay following transfection with B7H6 siRNA, negative siRNA. (C) Annexin V/PI staining and flow cytometry were used to examine the apoptosis rate following transfection with B7H6 siRNA. ${ }^{*} \mathrm{P}<0.05$ vs. negative siRNA. B7H6, natural killer cell cytotoxicity receptor 3 ligand 1 ; si, small interfering; PI, propidium iodide.
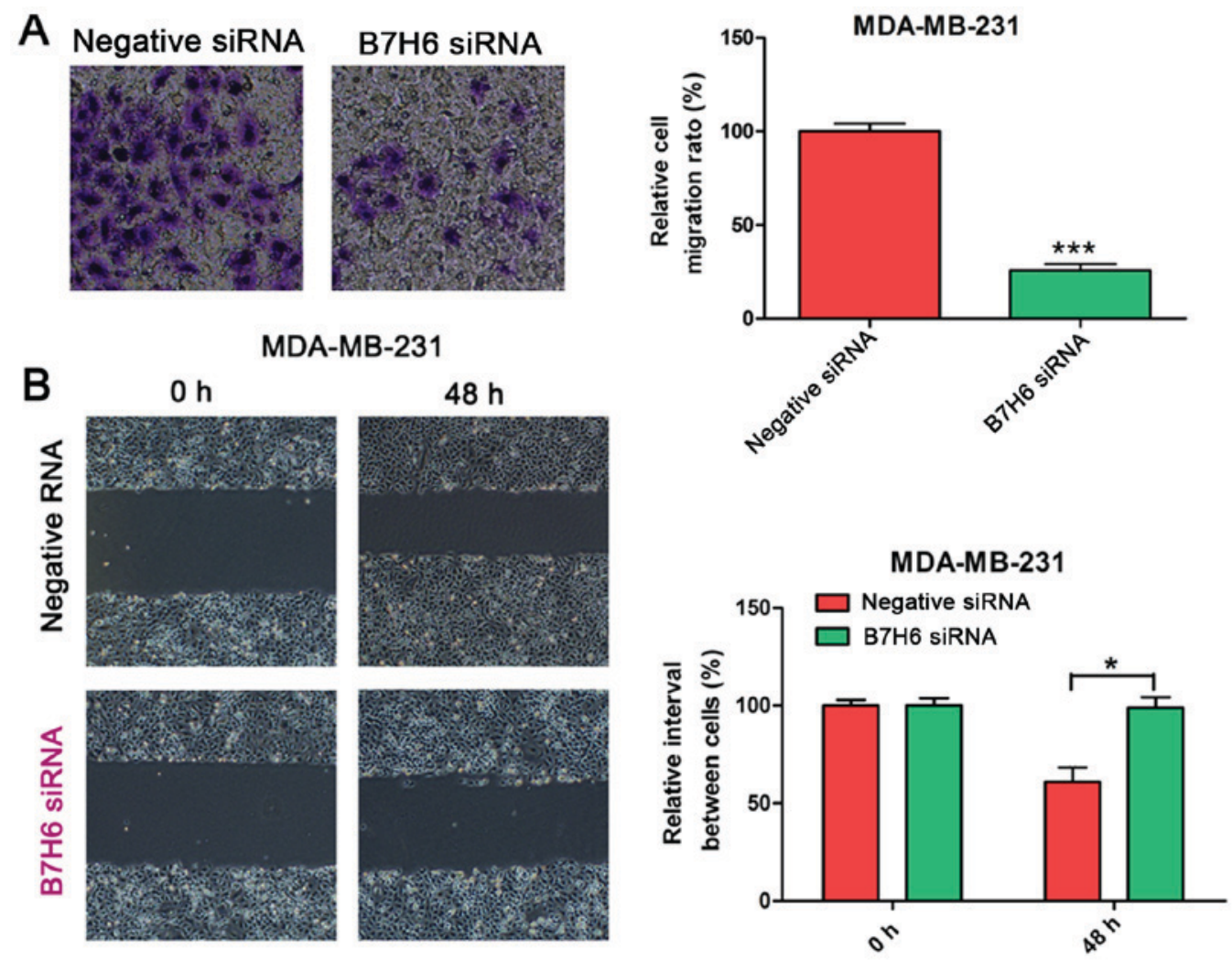

Figure 3. Effects of B7H6 knockdown on MDA-MB-231 cell migration ability. Cell migration ability was determined by (A) Transwell assay and (B) wound-healing assay in MDA-MB-231 cells transfected with B7H6 siRNA. Scrambled siRNA was used as the negative control. ${ }^{*} \mathrm{P}<0.05$ and ${ }^{* * * *} \mathrm{P}<0.001$ vs. negative siRNA. B7H6, natural killer cell cytotoxicity receptor 3 ligand $1 ;$ si, small interfering.

EGFR (cetuximab) was designed to treat breast cancer (23). Currently, the therapeutic strategies for TNBC primarily include surgery (breast conservation therapy and mastectomy) and chemotherapy $(24,25)$. But outcome still remains poor for 


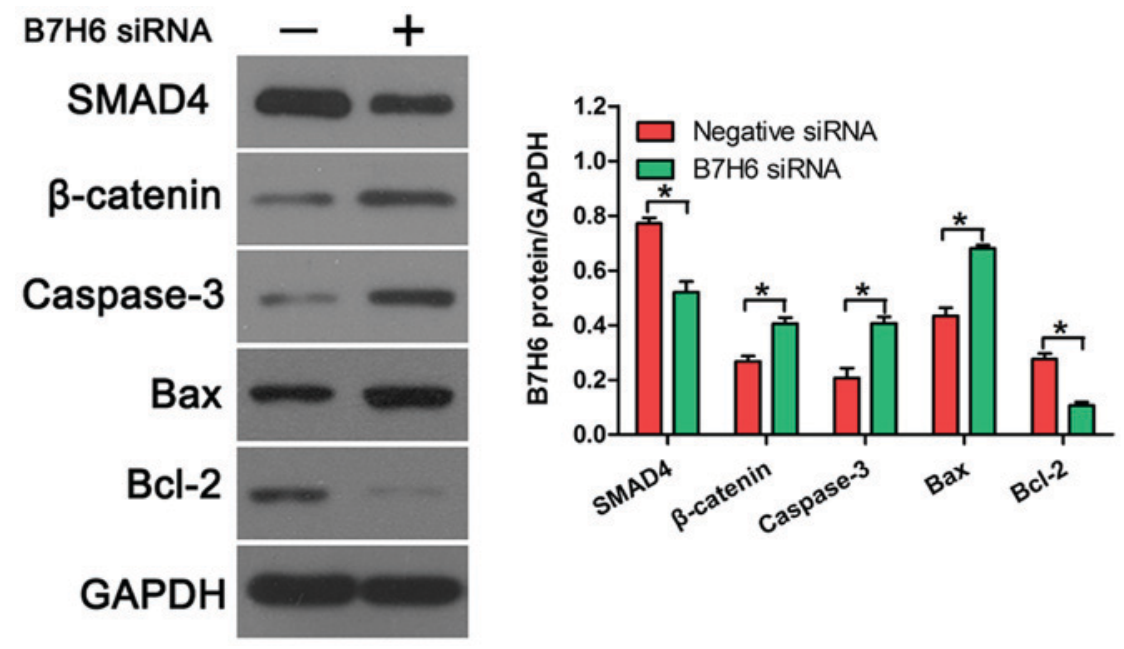

Figure 4. Effects of B7H6 knockdown on the expression of proliferation and apoptosis-related proteins. The expression levels of proliferation-related proteins (SMAD4 and $\beta$-Catenin) and apoptosis-related proteins (Bax, Bcl-2 and Caspase-3) were measured by western blot assay in MDA-MB-231 cells following transfection with B7H6 siRNA or negative control siRNA. "P<0.05 vs. negative siRNA control. B7H6, natural killer cell cytotoxicity receptor 3 ligand 1; SMAD4, SMAD family member 4; Bax, BCL2 associated X; Bcl-2, BCL2 apoptosis regulator; si, small interfering.

patients with TNBC and therefore targeted agents need to be investigated to explore novel treatments for TNBC.

The immune system has been demonstrated to crosstalk with tumor cells, and the aberrant expression of co-stimulatory or co-inhibitory molecules that belong to the B7 family has been considered to be involved in the suppression of antitumor immunity $(26,27)$. Therefore, the relationship between the B7 family and antitumor immunity is currently being studied to explore potentially novel effective therapeutic targets for various human cancers. Despite the undetermined function of $\mathrm{B} 7 \mathrm{H} 3$, experiments with tumor models tend to support a co-stimulatory role in antitumor immunity. For example, mouse P815 tumor cells transfected with B7H3 display enhanced immunogenicity (28). It was reported that $\mathrm{B} 7 \mathrm{H} 4$ is overexpressed in human BCs, and knockdown of $\mathrm{B} 7 \mathrm{H} 4$ by specific siRNA induced tumor cell apoptosis, suggesting a co-inhibitory role of B7H4 in BC cells (29). B7H6, a recently discovered member of the B7 family, has been reported to be expressed on tumors despite its absence in normal tissues, suggesting that it may be a potential target for cancer treatment (14). B7H6 was identified as a tumor-specific ligand for NCR3 (14). It has been reported that B7H6 mRNA expression is upregulated in leukemia and gastrointestinal stromal tumors and associated with clinical observations of NCR3-mediated immunosurveillance in these tumors $(30,31)$. However, the mechanism by which B7H6 affects tumor progression has not been identified completely in TNBC cells. Previously, it has been reported that several types of primary human tumors, including lymphoma, leukemia, ovarian cancer, breast cancer, renal cell carcinoma, various sarcomas and brain tumors, express high levels of B7H6 mRNA (32).

To investigate the exact role of B7H6 in BC first the expression levels of B7H6 were examined in several BC cell lines. The results demonstrated that B7H6 was highly expressed in the TNBC cell lines compared with non-TNBC and normal human mammary epithelial cell lines. To determine the function of B7H6 in TNBC cells, cell viability, apoptosis, and migration were measured following B7H6 knockdown in TNBC cells. The results demonstrated that B7H6 promoted TNBC cell proliferation and migration, and inhibited apoptosis.

Previous studies have demonstrated that SMAD4 is a tumor suppressor involved in the proliferation of many human cancers, including gastric, colorectal, and breast cancers (33-35). SMAD4 suppresses invasiveness and mediates reversion of SW480 cells from a mesenchymal-like to a polarized epithelial phenotype, with features of enterocyte-like differentiation (36). Therefore, the present study investigated whether B7H6 could regulate the production of SMAD4, and western blot analysis revealed that B7H6 knockdown decreased the expression of SMAD4. Because SMAD4 was reported to prevent tumor progression by diminishing the $\beta$-catenin signal, production of $\beta$-catenin was also measured and revealed to be upregulated following B7H6 knockdown in TNBC cells. Furthermore, the production of several apoptosis-related proteins was examined in B7H6-knockdown TNBC cells, and the results demonstrated that capase- 3 and Bax were upregulated, while Bcl-2 was downregulated. These data further confirmed the inhibitory effects of B7H6 in TNBC cells. The present results suggest that B7H6 might have important roles in the regulation of cell proliferation, apoptosis and migration in TNBC cells.

\section{Acknowledgements}

Not applicable.

\section{Funding}

No funding was received.

\section{Availability of data and materials}

All data generated or analyzed during this study are included in this published article. 


\section{Authors' contributions}

BZ and SS designed the study. JS, XY and JL performed the experiments. YY and FY analyzed the data. BZ wrote the manuscript. All authors have read and approved the final version of the manuscript.

\section{Ethics approval and consent to participate}

Not applicable.

\section{Consent for publication}

Not applicable.

\section{Competing interests}

The authors declare that they have no competing interests.

\section{References}

1. Kumar P and Aggarwal R: An overview of triple-negative breast cancer. Arch Gynecol Obstet 293: 247-269, 2016.

2. Boyle P: The globalisation of cancer. Lancet 368: 629-630, 2006.

3. Autier P, Boniol M, LaVecchia C, Vatten L, Gavin A, Héry C and Heanue M: Disparities in breast cancer mortality trends between 30 European countries: Retrospective trend analysis of WHO mortality database. Br Med J 341: c3620, 2010.

4. Bauer KR, Brown M, Cress RD, Parise CA and Caggiano V: Descriptive analysis of estrogen receptor (ER)-negative, progesterone receptor (PR)-negative, and HER2-negative invasive breast cancer, the so-called triple-negative phenotype: A population-based study from the California cancer Registry. Cancer 109: 1721-1728, 2007.

5. Carey LA, Dees EC, Sawyer L, Gatti L, Moore DT, Collichio F, Ollila DW, Sartor CI, Graham ML and Perou CM: The triple negative paradox: Primary tumor chemosensitivity of breast cancer subtypes. Clin Cancer Res 13: 2329-2334, 2007.

6. Sotiriou C and Pusztai L: Gene-expression signatures in breast cancer. N Engl J Med 360: 790-800, 2009.

7. Kreike B, van Kouwenhove M, Horlings H, Weigelt B, Peterse H, Bartelink $\mathrm{H}$ and van de Vijver MJ: Gene expression profiling and histopathological characterization of triple-negative/basal-like breast carcinomas. Breast Cancer Res 9: R65, 2007.

8. Foulkes WD, Smith IE and Reis-Filho JS: Triple-negative breast cancer. N Engl J Med 363: 1938-1948, 2010.

9. Krishnamurthy S, Poornima R, Challa VR and Goud YG: Triple negative breast cancer-our experience and review. Indian J Surg Oncol 3: 12-16, 2012.

10. Sethi S, Sarkar FH, Ahmed Q, Bandyopadhyay S, Nahleh ZA, Semaan A, Sakr W, Munkarah A and Ali-Fehmi R: Molecular markers of epithelial-to-mesenchymal transition are associated with tumor aggressiveness in breast carcinoma. Transl Oncol 4: 222-226, 2011.

11. Podo F, Buydens LM, Degani H, Hilhorst R, Klipp E, Gribbestad IS, Van Huffel S, van Laarhoven HW, Luts J, Monleon D, et al: Triple-negative breast cancer: Present challenges and new perspectives. Mol Oncol 4: 209-229, 2010.

12. Collins M, Ling V and Carreno BM: The B7 family of immune-regulatory ligands. Genome Biol 6: 223, 2005.

13. Flajnik MF, Tlapakova T, Criscitiello MF, Krylov V and Ohta Y: Evolution of the B7 family: Co-evolution of B7H6 and NKp30, identification of a new B7 family member, B7H7, and of B7's historical relationship with the MHC. Immunogenetics 64: 571-590, 2012.

14. Brandt CS, Baratin M, Yi EC, Kennedy J, Gao Z, Fox B, Haldeman B, Ostrander CD, Kaifu T, Chabannon C, et al: The B7 family member B7-H6 is a tumor cell ligand for the activating natural killer cell receptor NKp30 in humans. J Exp Med 206 . 1495-1503, 2009.

15. Wu MR, Zhang T, Gacerez AT, Coupet TA, DeMars LR and Sentman CL: B7H6-specific bispecific T cell engagers lead to tumor elimination and host antitumor immunity. J Immunol 194: $5305-5311,2015$
16. Wang G, Ye Y, Yang X,Liao H,ZhaoCandLiang S: Expression-based in silico screening of candidate therapeutic compounds for lung adenocarcinoma. PLoS One 6: e14573, 2011.

17. Elias AD: Triple-negative breast cancer: A short review. Am J Clin Oncol 33: 637-645, 2010.

18. Herold CI and Anders CK: New targets for triple-negative breast cancer. Oncology (Williston Park) 27: 846-854, 2013.

19. Miller K, Wang M, Gralow J, Dickler M, Cobleigh M, Perez EA, Shenkier T, Cella D and Davidson NE: Paclitaxel plus bevacizumab versus paclitaxel alone for metastatic breast cancer. N Engl J Med 357: 2666-2676, 2007.

20. Siziopikou KP and Cobteigh M: The basal subtype of breast carcinomas may represent the group of breast tumors that could benefit from EGFR-targeted therapies. Breast 16: 104-107, 2007.

21. Salazar N, Muñoz D, Kallifatidis G, Singh RK, Jordà M and Lokeshwar BL: The chemokine receptor CXCR7 interacts with EGFR to promote breast cancer cell proliferation. Mol Cancer 13: $198,2014$.

22. Cui W, Zhang S, Shan C, Zhou L and Zhou Z: microRNA-133a regulates the cell cycle and proliferation of breast cancer cells by targeting epidermal growth factor receptor through the EGFR/Akt signaling pathway. FEBS J 280: 3962-3974, 2013.

23. Carey LA, Rugo HS, Marcom PK, Mayer EL, Esteva FJ, Ma CX, Liu MC, Storniolo AM, Rimawi MF, Forero-Torres A, et al: TBCRC 001: Randomized phase II study of cetuximab in combination with carboplatin in stage IV triple-negative breast cancer. J Clin Oncol 30: 2615-2623, 2012.

24. Freedman GM, Anderson PR, Li T and Nicolaou N: Locoregional recurrence of triple-negative breast cancer after breast-conserving surgery and radiation. Cancer 115: 946-951, 2009.

25. Voduc KD, Cheang MC, Tyldesley S, Gelmon K, Nielsen TO and Kennecke H: Breast cancer subtypes and the risk of local and regional relapse. J Clin Oncol 28: 1684-1691, 2010.

26. Hanahan D and Weinberg RA: Hallmarks of cancer: The next generation. Cell 144: 646-674, 2011.

27. Leung J and Suh WK: The CD28-B7 family in anti-tumor immunity: Emerging concepts in cancer immunotherapy. Immune Netw 14: 265-276, 2014.

28. Luo L, Chapoval AI, Flies DB, Zhu G, Hirano F, Wang S, Lau JS, Dong H, Tamada K, Flies AS, et al: B7-H3 enhances tumor immunity in vivo by costimulating rapid clonal expansion of antigen-specific CD8+ cytolytic T cells. J Immunol 173: 5445-5450, 2004.

29. Salceda S, Tang T, Kmet M, Munteanu A, Ghosh M, Macina R, Liu W, Pilkington G and Papkoff J: The immunomodulatory protein $\mathrm{B} 7-\mathrm{H} 4$ is overexpressed in breast and ovarian cancers and promotes epithelial cell transformation. Exp Cell Res 306: 128-141, 2005.

30. Delahaye NF, Rusakiewicz S, Martins I, Ménard C, Roux S, Lyonnet L, Paul P, Sarabi M, Chaput N, Semeraro M, et al: Alternatively spliced NKp30 isoforms affect the prognosis of gastrointestinal stromal tumors. Nat Med 17: 700-707, 2011.

31. Fauriat C, Just-Landi S, Mallet F, Arnoulet C, Sainty D, Olive D and Costello RT: Deficient expression of NCR in NK cells from acute myeloid leukemia: Evolution during leukemia treatment and impact of leukemia cells in NCRdull phenotype induction. Blood 109: 323-330, 2007.

32. Wu MR, Zhang T, DeMars LR and Sentman CL: B7H6-specific chimeric antigen receptors lead to tumor elimination and host antitumor immunity. Gene Ther 22: 675-684, 2015.

33. Miyaki M, Iijima T, Konishi M, Sakai K, Ishii A, Yasuno M, Hishima T, Koike M, Shitara N, Iwama T, et al: Higher frequency of Smad4 gene mutation in human colorectal cancer with distant metastasis. Oncogene 18: 3098-3103, 1999.

34. Kundu J, Wahab SM, Kundu JK, Choi YL, Erkin OC, Lee HS, Park SG and Shin YK: Tobl induces apoptosis and inhibits proliferation, migration and invasion of gastric cancer cells by activating Smad4 and inhibiting betacatenin signaling. Int J Oncol 41: 839-848, 2012.

35. Deckers M, van Dinther M, Buijs J, Que I, Löwik C, van der Pluijm G and ten Dijke P: The tumor suppressor Smad4 is required for transforming growth factor beta-induced epithelial to mesenchymal transition and bone metastasis of breast cancer cells. Cancer Res 66: 2202-2209, 2006.

36. Pohl M, Radacz Y, Pawlik N, Schoeneck A, Baldus SE, Munding J, Schmiegel W, Schwarte-Waldhoff I and Reinacher-Schick A: SMAD4 mediates mesenchymal-epithelial reversion in SW480 colon carcinoma cells. Anticancer Res 30: 2603-2613, 2010. 